\title{
APLICAÇÃO DE TÉCNICAS RADIOGRÁFICAS PARA O DIAGNÓSTICO DIFERENCIAL DE TONSILOLITO
}

\section{APPLICATION OF RADIOGRAPHIC TECHNIQUES FOR DIFFERENTIAL DIAGNOSIS OF TONSILLOLITH}

\author{
Maria Luiza dos Anjos Pontual * \\ Andréa dos Anjos Pontual * \\ Márcia Maria Fonseca da Silveira ** \\ Marcelo Tarcísio Martins *** \\ Karina Lopes Devito $\cdots \cdot$
}

\begin{abstract}
RESUMO
A radiografia panorâmica é um dos exames radiográficos mais utilizados na rotina odontológica, existindo grande possibilidade do cirurgião-dentista se deparar com achados radiográficos. Dentre estes, destacam-se as calcificações patológicas encontradas na região de ramo mandibular, tais como sialolito, tonsilolito, calcificações de linfonodos e de vasos sanguíneos. As calcificações patológicas podem possuir aspecto radiográfico semelhante em uma radiografia panorâmica, sendo necessária a complementação com outras técnicas radiográficas a fim de auxiliar no diagnóstico diferencial. Neste trabalho é apresentada uma revisão da literatura a respeito das características radiográficas das calcificações de tecidos moles mais comuns da clínica odontológica. Além disso, é demonstrada a aplicação de técnicas radiográficas no diagnóstico diferencial de tonsilolitos. Diante do exposto, concluiu-se que a associação de técnicas extrabucais é uma importante ferramenta para o preciso diagnóstico de sialolitos, escleroses ósseas e tonsilolitos.

DESCRITORES: Radiografia panorâmica • Calcinose • Glândulas salivares • Dor facial.
\end{abstract}

\section{ABSTRACT}

Panoramic radiography is one of the radiographic exams most used in routine dentistry, and there is great possibility of the dentist encountering unexpected radiographic findings. Among these, the following are outstanding: pathologic calcifications found in the region of the mandibular ramus, such as sialolith, tonsillolith, lymph node and blood vessel calcifications. Pathologic calcifications may have a similar radiographic appearance in a panoramic radiograph, so it is necessary the complementation with other radiographic techniques to aid the differential diagnosis. In this work is presented a literature review about the radiographic characteristics of the more common soft tissue calcifications of the dental clinical. Moreover, it is demonstrated the application of radiographic techniques in the diagnostic differential of tonsilloliths. In view of the above, it was concluded that the association of extra-oral techniques is an important tool for the precise diagnosis of sialoliths, osseous sclerosis and tonsilloliths.

DESCRIPTORS: Radiography, panoramic • Calcinosis • Salivary glands $\bullet$ Facial pain.

\footnotetext{
* Professora Adjunta da Faculdade de Odontologia da Universidade Federal da Paraíba (UFPB), João Pessoa, Paraíba.

** Professora Adjunta da Faculdade de Odontologia da Universidade de Pernambuco (UPE), Recife, Pernambuco.

*** Mestrando do Programa de Pós-Graduação em Clínica Odontológica da Universidade Federal de Juiz de Fora (UFJF), Juiz de Fora, Minas Gerais

**** Professora Adjunta da Faculdade de Odontologia da Universidade Federal de Juiz de Fora (UFJF), Juiz de Fora, Minas Gerais
} 


\section{N T RO DUÇÃ O}

São consideradas calcificações patológicas toda deposição anormal de sais de cálcio, juntamente com quantidades menores de ferro, magnésio e outros minerais. Quando estas deposições acontecem em tecidos moles sadios ou degenerados são denominadas de calcificações distróficas (Almog et al. ${ }^{1}, 2000$ ). Friedlander e Lande $^{2}$, 1981) encontraram $2 \%$ de evidências de calcificações patológicas na região cervical quando analisaram 1000 radiografias panorâmicas de rotina, de pacientes com idade variando entre 50 e 75 anos. As calcificações podem estar presentes em vasos sanguíneos, glândulas salivares, linfonodos e tonsilas. Além dessas calcificações, estruturas anatômicas também podem se apresentar calcificadas, tais como a cartilagem tritícea e o ligamento estilo-hióideo. Em muitos casos, a localização e a morfologia típica são as únicas ferramentas para auxiliar no diagnóstico diferencial dessas calcificações em radiografias panorâmicas. Entretanto, por vezes as calcificações podem apresentar localização e aspecto radiográfico semelhantes, dificultando a diferenciação entre elas (Carter $\left.{ }^{3}, 2000\right)$.

Os tonsilolitos correspondem a concreções presentes nas tonsilas, sendo geralmente associados à história de tonsilolite crônica. Segundo Revel et al. ${ }^{4}, 1998$ a patogênese do tonsilolito é muito controversa e autores como Cooper et al. ${ }^{5}$, 1983 e Pruet e Duplan ${ }^{6}, 1987$ acreditam que essas calcificações resultam de uma tonsilolite não curada e que a presença de células e bactérias mortas serve como um nicho para o início da calcificação. Já Mishenkin e Shtil 71965 sugeriram que a formação do cálculo tonsilar pode ser causada pela estase salivar nos ductos eferentes das glândulas salivares acessórias.

O tonsilolito pode ocorrer em qualquer faixa etária, com idade média de 40 anos, não apresentando predileção por sexo (Neshat et al. ${ }^{8}, 2001$; Pruet e Duplan ${ }^{6}$, 1987). Quando pequenos, geralmente são assintomáticos, mas nos casos de tamanhos maiores o paciente pode referir dor, inchaço, infecção, halitose, disfagia, inflamação crônica e sensação de corpo estranho na laringe (Cooper et al. ${ }^{5}, 1983$;
Neshat et al. $\left.{ }^{8}, 2001\right)$.

Clinicamente, os tonsilolitos podem ser detectados pela palpação da fossa tonsilar (Hoffman ${ }^{9}$, 1978), assemelhando-se a um tumor (Revel et al. ${ }^{4}, 1998$ ). Ao exame radiográfico, podem ser observados como radiopacidades únicas ou múltiplas, de tamanhos variados e de formatos esférico, ovoide ou irregular (Cooper et al. ${ }^{5}, 1983$; Neshat et al. ${ }^{8}, 2001$ ) e podem ser de difícil diagnóstico por assemelharem-se a dentes ectópicos (Revel et al. ${ }^{4}, 1998$; Von Arx e $\mathrm{Carr}^{10}$, 1988), calcificações orofaringeanas e estruturas anatômicas (Aspestrand e Kolbenstvedt ${ }^{11}$, 1987; Revel et al. ${ }^{4}, 1998$; Sezer et al. ${ }^{12}$, 2003). Na radiografia panorâmica, os tonsilolitos apresentam-se como estruturas radiopacas sobrepostas na porção medial no ramo ascendente da mandíbula.

Na presença de dor, infecção e edema, sua remoção torna-se necessária, podendo ser realizada por meio de leve pressão manual ou curetagem sob anestesia local e, quando necessário, faz-se uso de incisão (Neshat et al. ${ }^{8}, 2001$ ).

Na sequência será apresentada uma revisão da literatura enfatizando os aspectos radiográficos dos tonsilolitos e das principais entidades patológicas que devem ser consideradas no seu diagnóstico diferencial. Além disso, serão apresentadas calcificações de localização e formato semelhantes, projetadas na região de ramo mandibular em radiografias panorâmicas e, a partir dessas imagens, será demonstrada a aplicação de técnicas radiográficas extrabucais como auxiliares no diagnóstico dessas calcificações.

\section{REVISÃO DE LITERATURA}

$\mathrm{Na}$ região cervical pode haver ocorrência de sialolitos, tonsilolitos, calcificações de linfonodos e de vasos sanguíneos (Manzi et al. ${ }^{13}, 2001$; Monsour e Mendoza ${ }^{14}, 1990$; Pontual et al. ${ }^{15}$, 2003). Para um correto tratamento, essas calcificações devem ser diferenciadas entre si, de estruturas anatômicas e também de outras estruturas passíveis de calcificação, como do ligamento estilo-hióideo (Carter ${ }^{3}, 2000$; Pontual et al. ${ }^{15}$, 2003; Monsour e Young $\left.{ }^{16}, 1986\right)$. As distinções entre as calcificações podem ser realizadas de ma-
PONTUAL MLA

pontual aA

SILVEIRA MMF MARTINS MT

DEVITO KL

APLICAÇÃO

DE TÉCNICAS

RADIOGRÁFICAS

PARA O

DIAGNÓSTICO

DIFERENCIAL DE

TONSILOLITO
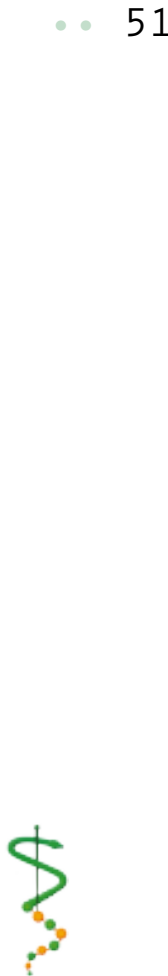

REVISTA DE

ODONTOLOGIA DA

UN I VERS I DADE

CIDADE DE SÃO

PAULO

$2010 ; 22(1): 50-5$, JAN - ABR 
PONTUAL MLA

PONTUAL AA

SILVEIRA MMF

MARTINS MT

DEVITO KL

APLICAÇÃO

DE TÉCNICAS

RADIOGRÁFICAS

PARA 0

DIAGNÓSTICO

DIFERENCIAL DE

TONSILOLITO

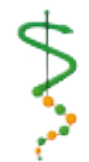

REVISTA DE

ODONTOLOGIA DA

UNI VERSIDADE

CIDADE DE SÃO

PAULO

$2010 ; 22(1): 50-5$,

$J A N-A B R$ neira confiável por meio da associação de critérios radiográficos e clínicos (Cohen et al. $\left.{ }^{17}, 2002\right)$.

A região de ramo mandibular, em radiografias panorâmicas, pode apresentar imagens de radiopacidades intraósseas e sobreposições de radiopacidades mediais e laterais ao ramo. Dentre elas, podemse citar as calcificações do ligamento estilo-hióideo, de vasos, de linfonodos, de glândulas salivares e das tonsilas. A calcificação do ligamento estilo-hióideo é facilmente diferenciada por apresentar-se como a mineralização que geralmente envolve todo o ligamento estilo-hióideo, região compreendida entre a porção inferior do processo estiloide e o corno menor do osso hioide (Almog et al. ${ }^{1}, 2000$; Pontual et al. ${ }^{15}$, 2003). Além da diferente extensão, a calcificação do ligamento estilo-hióideo possui formatos diferentes, sendo classificada por Langlais et al. ${ }^{17}, 1986$ como: alongado, segmentado ou pseudoarticulado.

As calcificações distróficas encontradas em vasos são denominadas de flebólitos e devem-se a malformações arteriovenosas. Radiograficamente apresentam-se menores que os cálculos salivares, geralmente múltiplas, circulares e frequentemente com radiopacidade concêntrica e anéis radiolucentes (Almog et al. ${ }^{1}, 2000$ ), sendo mais comuns na região submandibular (Keathley et al. ${ }^{20}$, 1983).

As calcificações dos linfonodos podem indicar a ocorrência de um processo infeccioso crônico anterior, comum em casos de tuberculose crônica (Williams e Douglas-Jones ${ }^{19}$, 1995). Normalmente são vistas na região posterior ao ramo ou próximas ao ângulo da mandíbula, estando algumas vezes sobrepostas a este osso ou sobre o ápice de um elemento dentário inferior (Katz et al. ${ }^{20}, 1989$ ). Ainda, são quase sempre unilaterais, podendo ser únicas, múltiplas ou em cadeia, dando uma configuração de corrente (Almog et al. ${ }^{1}, 2000$; Katz et al. ${ }^{21}$, 1989).

Já os sialolitos da glândula parótida apresentam localização bastante semeIhante ao tonsilolito na radiografia panorâmica, ficando projetados no terço médio do ramo mandibular. Para auxílio do diagnóstico diferencial, pode-se lançar mão da associação de técnicas radiográficas que revelem a localização da calcificação no sentido látero-medial. Na Figura 1A, observam-se radiopacidades ovoides projetadas no ramo mandibular direito, sugerindo sialolito de glândula parótida, esclerose óssea ou tonsilolito. Para auxiliar no diagnóstico diferencial, foi realizado um

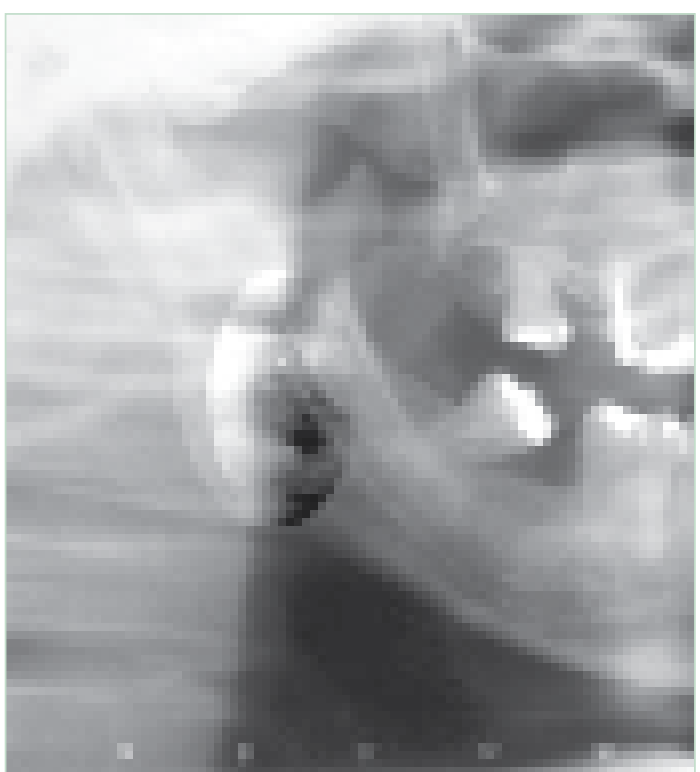

Figura 1. A Lado direito de uma radiografia panorâmica apresentando radiopacidades projetadas no ramo mandibular.

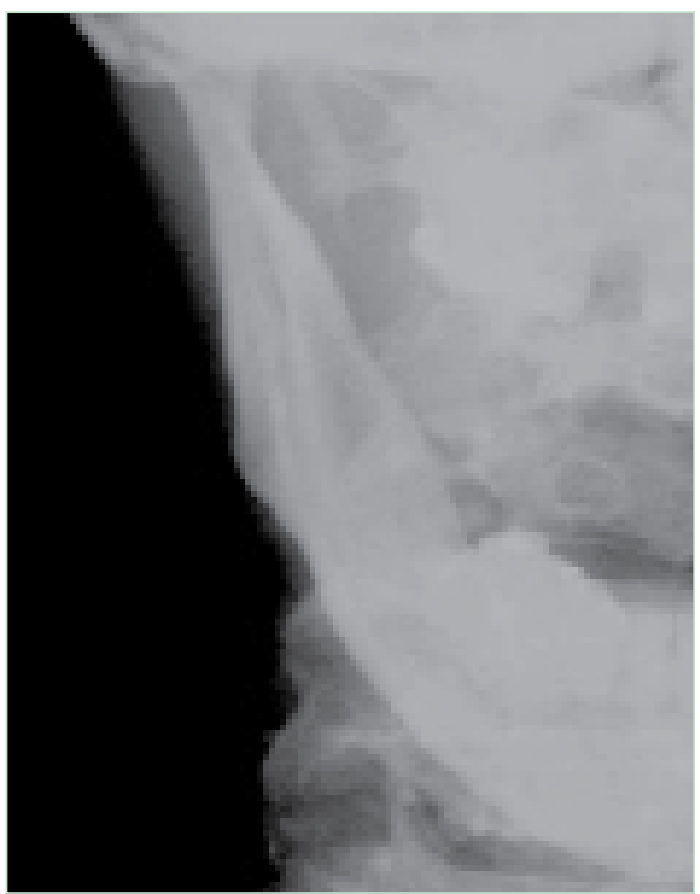

Figura 1. B Lado direito de uma radiografia póstero-anterior de mandíbula em boca aberta na qual se visualizam radiopacidades múltiplas na região correspondente à tonsila direita. 
exame radiográfico póstero-anterior com a boca aberta (Figura 1B) para determinar a localização no sentido médio-lateral dessas calcificações. Esse exame não evidenciou nenhuma calcificação no ramo da mandíbula, descartando a hipótese de esclerose óssea. Além disso, observou-se que a imagem radiopaca encontrava-se medialmente ao ramo mandibular, na região correspondente à tonsila direita, confirmando a hipótese de tonsilolito.

Pode-se também associar outra técnica ao exame radiográfico panorâmico, como a técnica lateral oblíqua da região de ramo e ângulo de mandíbula. Na Figura $2 \mathrm{~A}$, visualizam-se radiopacidades múltiplas de formato irregular, projetadas no ramo mandibular direito, acima do ângulo mandibular, sugestivas de tonsilolitos, esclerose óssea ou sialolitos da glândula parótida. Já na radiografia lateral oblíqua de ramo e ângulo de mandíbula, essas radiopacidades são projetadas superiormente ao demonstrado na radiografia panorâmica (Figura 2B). Portanto, pode-se concluir que as calcificações seriam de estruturas com uma localização mais medial que a da glândula parótida, indicando tratar-se de tonsilolitos. A hipótese de esclerose ós-

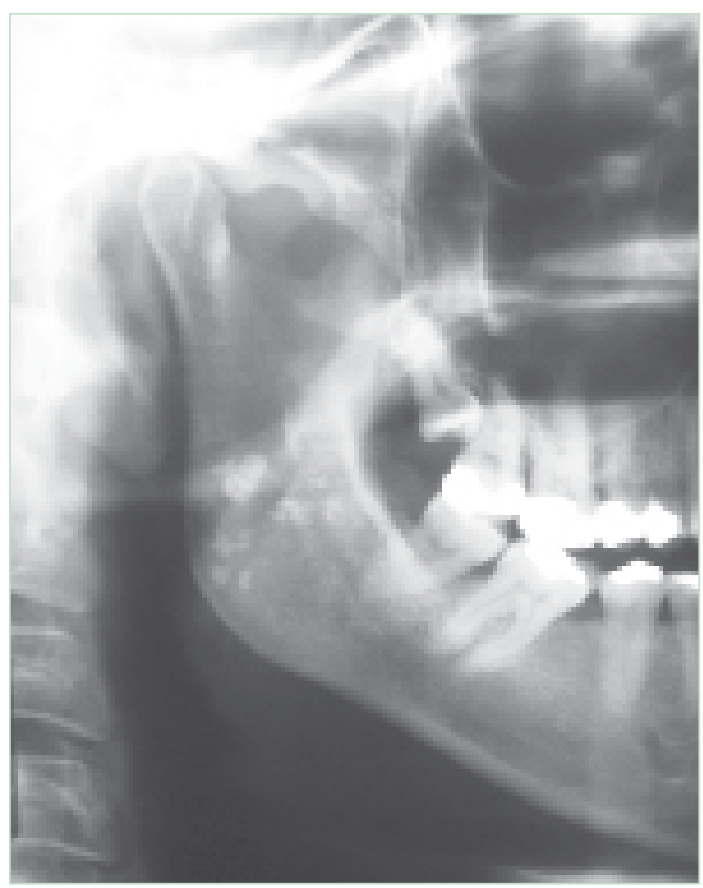

Figura 2. A Observam-se no lado direito de uma radiografia panorâmica radiopacidades projetadas na região de ramo mandibular. sea é descartada, pois, se assim fosse, não haveria mudança tão evidente na posição das radiopacidades.

A variação da posição do objeto nas diferentes incidências radiográficas é muito utilizada para identificar a localização das calcificações em tecido mole. Ademais, é um método valioso na definição do diagnóstico de tonsilolitos, devido à semelhança da localização de sialolitos da glândula parótida e escleroses intraósseas, visualizadas na mesma região em radiografias panorâmicas.

Anatomicamente, a glândula parótida encontra-se na região lateral ao ramo, enquanto que as tonsilas encontram-se na região medial. Nos casos apresentados, foram utilizadas técnicas radiográficas conjugadas para a localização médio-lateral das calcificações, o que possibilitou a identificação das radiopacidades na região medial em relação ao ramo, região correspondente à tonsila.

\section{CONCLUSÃO}

A associação de técnicas extrabucais é uma importante ferramenta para o diagnóstico diferencial de sialolitos da glândula parótida, escleroses ósseas e tonsilolitos.

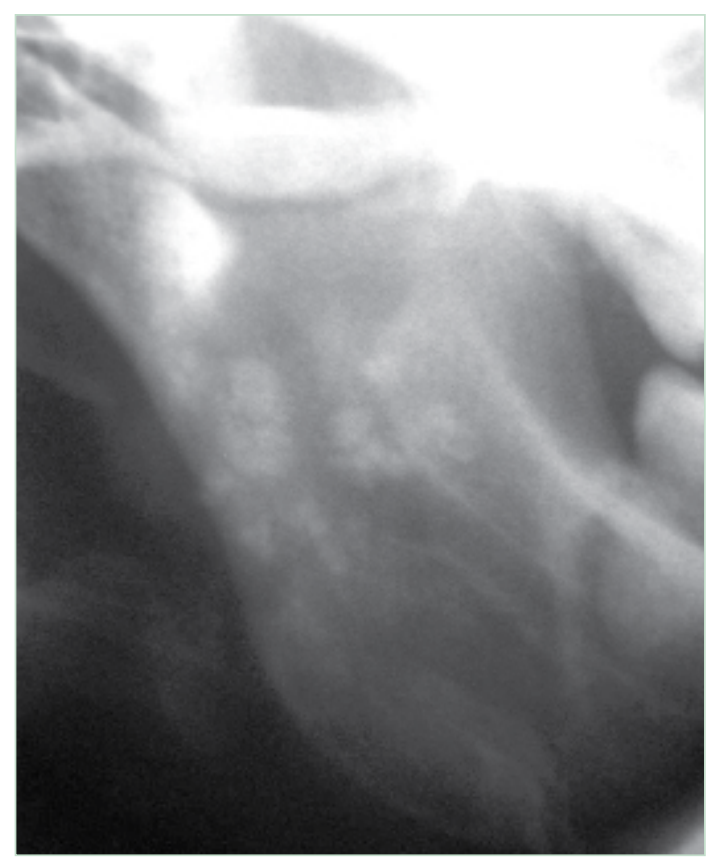

Figura 2. B Na radiografia lateral oblíqua de ramo e ângulo mandibular direito, essas radiopacidades são projetadas mais superiormente, sugerindo localização medial.
PONTUAL MLA

pontual aA

SILVEIRA MMF

MARTINS MT

DEVITO KL

APLICAÇÃO

DE TÉCNICAS

RADIOGRÁFICAS

PARA O

DIAGNÓSTICO

DIFERENCIAL DE

TONSILOLITO

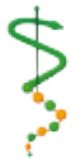

REVISTA DE ODONTOLOGIA DA UN I VERS I DADE CIDADE DE SÃO PAULO

$2010 ; 22(1) ; 50-5$, JAN - ABR 
PONTUAL MLA

PONTUAL AA

SILVEIRA MMF

MARTINS MT

DEVITO KL

APLICAÇÃO

DE TÉCNICAS

RADIOGRÁFICAS

PARA 0

DIAGNÓSTICO

DIFERENCIAL DE

TONSILOLITO

54

\section{REFERÊNCIAS}

1. Almog DM, Tsimidis K, Moss ME, Gottlieb RH, Carter LC. Evaluation of a training program for detection of carotid artery calcifications on panoramic radiographs. Oral Surg Oral Med Oral Pathol Oral Radiol Endod 2000 Jul; 90(1):111-7.

2. Friedlander $\mathrm{AH}$, Lande $\mathrm{A}$. Panoramic radiographic identification of carotid arterial plaques. Oral Surg Oral Med Oral Pathol 1981 Jul; 52(1):102-4.

3. Carter LC. Discrimination between calcified triticeous cartilage and calcified carotid atheroma on panoramic radiography. Oral Surg Oral Med Oral Pathol Oral Radio Endod 2000 Jul; 90(1):108-10.

4. Revel MP, Bely N, Laccourreye O, Naudo P, Hartl D, Brasnu D. Giant tonsillolith. Ann Otol Rhinol Laryngol 1998 Mar; 107(3): 262-3.

5. Cooper MM, Steinberg JJ, Lastra M, Antopol S. Tonsillar calculi. Report of a case and review of the literature. Oral Surg Oral Med Oral Pathol 1983 Mar; 55(3): 239-43.

6. Pruet CW, Duplan DA. Tonsil concretions and tonsilloliths. Otlaryngol Clin North Am 1987 May; 20(2): 305-9.

7. Mishenkin NV, Shtil AL. Calculi of palatine tonsils and causes of their formation. Vestn Otorinolaringol 1965 Mar-Apr; 27: 110-2.

8. Neshat K, Penna KJ, Shah DH. Tonsillolith: a case report. J Oral Maxillofac Surg 2001 Jun; 59(6): 692-3.

9. Hoffman H. Tonsillolith. Oral Surg Oral Med Oral Pathol 1978 Apr; 45(4): 657-8.

10. Von Arx D, Carr RJ. Displaced tooth mimicking a tonsillolith. J Laryngol Otol 1988 Jul; 102(7): 652-3.

11. Aspestrand F, Kolbenstvedt A. Calcifications of the palatine tonsillary region: CT demonstration. Radiology 1987 Nov; 165(2): 479-80.

12. Sezer B, Tugsel Z, Bilgen C. An unusual tonsillolith. Oral Surg Oral Med Oral Pathol Oral Radiol Endod 2003 Apr; 95(4): 471-3.

13. Manzi FR, Tuji FM, Haiter Neto F, Bóscolo FN. Radiografia panorâmica como meio auxiliar na identificação de pacientes com risco de AVC. Rev Assoc Paul Cir Dent 2001; 55(2): 131-3.

14. Monsour PA, Mendoza AR. Panoramic ghost images as an aid in the localization of soft tissue calcifications. Oral Surg Oral Med Oral Pathol 1990 Jun; 69(6): 748-56.

15. Pontual MLA, Martins MGBQ, Freire Filho FWV, Haiter Neto F, Moraes M. Diagnóstico diferencial das calcificações da região cervical - revisão da literatura. Rev Assoc Paul Cir Dent 2003; 57(6): 429-33.

16. Monsour PA, Young WG. Variability of the styloid process and stylohyoid ligament in panoramic radiographs. Oral Surg Oral Med Oral Pathol 1986 May; 61(5): 522-6.

17. Cohen SN, Friedlander AH, Jolly DA, Date L. Carotid calcification on panoramic radiographs: an important marker for vascular risk. Oral Surg Oral Med Oral Pathol Oral Radiol Endod 2002 Oct; 94(4): 510-4.

18. Langlais RP, Miles DA, Van Dis ML. Elongated and mineralized stylohyoid ligament complex: a proposed classification and report of a case of Eagle`s syndrome. Oral Surg Oral Med Oral Pathol 1986 May; 61(5): 527-32.

19. Keathley CJ, Campbell RL, Isbell JW. Arteriovenous malformation and associated phleboliths: Report of a case. Oral Surg Oral Med Oral Pathol 1983 Aug; 56(2):132-5.

20. Williams RG, Douglas-Jones T. Mycobacterium marches back. J Laryngol Otol 1995 
Jan; 109(1): 5-13.

21. Katz JO, Langlais RP, Underhill TE, Kimura K. Localization of paraoral soft tissue calcifications: the known object rule. Oral Surg Oral Med Oral Pathol 1989 Apr; 67(4): 459-63.

Recebido em: 11/03/2009

Aceito em: 01/10/2009
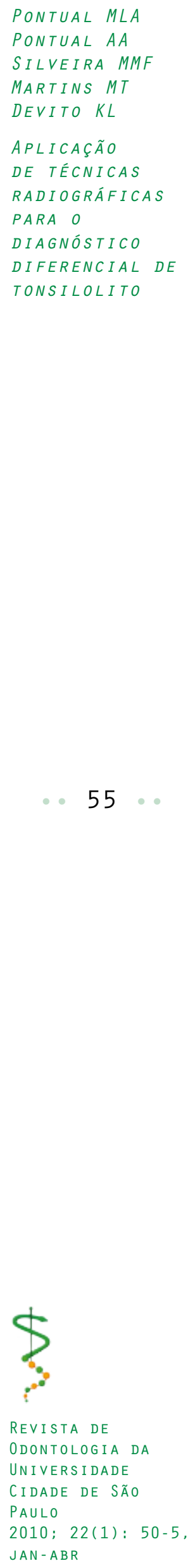